Europhysics Letters

PREPRINT

\title{
The distribution function of a semiflexible polymer and random walks with constraints
}

\author{
Semjon Stepanow ${ }^{1}$ and Gunter M. Schütz ${ }^{2}$ \\ 1 Martin-Luther-Universität Halle, Fachbereich Physik, D-06099 Halle, Germany \\ 2 Institut für Festkörperforschung, Forschungszentrum Jülich, D-52425 Jülich, Ger- \\ many
}

PACS. 36.20.-r - Macromolecules and polymer molecules.

PACS. 05.40-a - Fluctuation Phenomena, random processes, noise, and Brownian motion.

PACS. 03.65.Fd - Algebraic methods.

\begin{abstract}
In studying the end-to-end distribution function $G(r, N)$ of a worm like chain by using the propagator method we have established that the combinatorial problem of counting the paths contributing to $G(r, N)$ can be mapped onto the problem of random walks with constraints, which is closely related to the representation theory of the Temperley-Lieb algebra. By using this mapping we derive an exact expression of the Fourier-Laplace transform of the distribution function, $G(k, p)$, as a matrix element of an inverse of an infinite rank matrix. Using this result we also derived a recursion relation permitting to compute $G(k, p)$ directly. We present the results of the computation of $G(k, N)$ and its moments. The moments $\left\langle r^{2 n}\right\rangle$ of $G(r, N)$ can be calculated exactly by calculating the $(1,1)$ matrix element of $2 n$-th power of a truncated matrix of rank $n+1$.
\end{abstract}

The theory of flexible polymers is now understood [1]- [4]. Many polymer molecules have internal stiffness and cannot be modeled by the model of flexible molecules developed by Edwards. This is especially true for several important biopolymers such as actin, DNA, and microtubules [5]. Models of semiflexible polymers have also applications in different topics besides polymer physics [6]. If the chain length decreases, the chain stiffness becomes an important factor. A quantitative measure for the stiffness of the polymer is the persistence length $l_{p}$, which is the correlation length for the tangent-tangent correlation function along the polymer. Polymers with contour length $L$ much larger than $l_{p}$ are flexible and are described by using the tools of quantum mechanics and quantum field theory [1]- [1]. The standard coarse-graining model of a wormlike or a semiflexible polymer was proposed by Kratky and Porod [7]. A few first moments of $G(r, N)$ were computed in [8]- [10]. The literature on the computation of $G(r, N)$ and its moments can be found in the book by Yamakawa 11. For recent work see [12]- [14].

In this Letter we will study the problem of computation of the distribution function $G(r, N)$ of the end-to-end distance of a semiflexible polymer, which is described by Kratky-Porod model, by using the analogy of the worm like chain with the quantum rigid rotator in an external homogeneous field [3], 15] within the quantum mechanical propagator method [16].

(C) EDP Sciences 
The relation to the rigid rotator was used in the recent work [14]. Relating the combinatorics of counting the paths contributing to $G(r, N)$ to random walks with constraints and the Temperley-Lieb algebra [17], we have derived an exact formula for the Fourier-Laplace transform of the end-to-end distribution function, $G(k, p)$, as an inverse of an infinite rank matrix of a very simple structure, given by Eqs.(6-17). Using a truncated matrix of rank $n+1$ one obtains $n$ moments of $r^{2}$ exactly from the $(1,1)$ element of the $2 n$-th power of this matrix, i.e., by applying this matrix $2 n$ times to a canonical basis vector. Moreoever, this enables one to compute the distribution function, which describes $n$ moments of $r^{2}$ exactly and all higher moments approximately. The truncation corresponds to taking into account the intermediate states with the quantum number of the angular momentum $l \leq n$ (see below).

The Fourier transform of the distribution function of the end-to-end polymer distance of the Kratky-Porod model [7] $G(\mathbf{k}, L)=\int d^{3} R \exp \left(-i \mathbf{k}\left(\mathbf{R}-\mathbf{R}_{\mathbf{0}}\right)\right) G\left(\mathbf{R}-\mathbf{R}_{0}, L\right)$ is given by the path integral as follows

$$
G(\mathbf{k}, L)=\int D \mathbf{t}(s) \prod_{s} \delta\left(\mathbf{t}(s)^{2}-1\right) \exp \left(-i \mathbf{k} \int_{0}^{L} d s \mathbf{t}(s)-\frac{l_{p}}{2} \int_{0}^{L} d s\left(\frac{\partial \mathbf{t}(s)}{\partial s}\right)^{2}\right),
$$

where $l_{p}$ is the persistence length, and $\mathbf{t}(s)$ is the tangent vector at the point $s$ along the contour of the polymer. The product over $s$ in Eq.(1i) takes into account that the polymer chain is locally inextensible. If the polymer interacts with an external field, the potential energy $-\int_{0}^{L} d s V(\mathbf{r}(s))$ should be added in the exponential of Eq.(11). Notice that the representation of $G(k, L)$ by using the cumulants of the moments of the end-to-end distribution function $G(\mathbf{k}, L)=\exp \left(-\sum_{m=1}^{\infty} \int_{0}^{L} d s \mu_{2 m}(s)\left(\mathbf{k}^{2}\right)^{m}\right)$ permits to write the following differential equation for $G(\mathbf{R}, L), \partial G(\mathbf{R}, L) / \partial L-\sum_{m=1}^{\infty}(-1)^{m+1} \mu_{2 m}(L) \Delta_{\mathbf{R}}^{m} G=0$, where $\Delta=\nabla^{2}$ is the Laplace operator. The latter with the term associated with the external potential was used in [18] to describe the localization of a semiflexible polymer onto interfaces and surfaces.

The path integral (1) (without the term depending on $k$ ) corresponds to the diffusion of a particle on unit sphere, $|\mathbf{t}(s)|=1$. This problem is equivalent to the Euclidean rigid quantum rotator [3], 15. The Green's function of the rigid rotator or a particle on unit sphere fulfills the following equation

$$
\frac{\partial}{\partial L} P_{0}\left(\theta, \varphi, L ; \theta_{0}, \varphi_{0}, 0\right)-\frac{1}{2 l_{p}} \nabla_{\theta, \varphi}^{2} P_{0}=\delta(L) \delta\left(\Omega-\Omega_{0}\right),
$$

where $\Omega$ is the spheric angle associated with angles $\theta$, and $\varphi$, and $\delta\left(\Omega-\Omega_{0}\right)$ is a two dimensional delta function. Henceforth instead of the contour length $L$ we will use the number of statistical segments $N=L / l_{p}$. Notice that in Quantum Mechanics $N$ corresponds to the imaginary time $i t$. The solution for $P_{0}\left(\theta, \varphi, N ; \theta_{0}, \varphi_{0}, 0\right)$ is

$$
P_{0}\left(\theta, \varphi, N ; \theta_{0}, \varphi_{0}, 0\right)=\sum_{l, m} \exp \left(-\frac{l(l+1) N}{2}\right) Y_{l m}(\theta, \varphi) Y_{l m}^{*}\left(\theta_{0}, \varphi_{0}\right),
$$

where $Y_{l m}(\theta, \varphi)$ are the spherical harmonics. At a given $l, m$ takes the values $-l,-l+1, \ldots$, $l$. The quantity $P_{0}\left(\theta, \varphi, N ; \theta_{0}, \varphi_{0}, 0\right)$ is given by Eq.(1) with $\mathbf{k}=0$ and with the following boundary conditions for the path $\mathbf{t}(s)(0 \leq s \leq N): \mathbf{t}(N) \equiv(\theta, \varphi)$, and $\mathbf{t}(0) \equiv\left(\theta_{0}, \varphi_{0}\right)$.

We now will consider the Green's function $P\left(\theta, \varphi, N ; \theta_{0}, \varphi_{0}, 0\right)$ associated with Eq.(11). The differential equation for $P$ is

$$
\frac{\partial}{\partial N} P\left(\theta, \varphi, N ; \theta_{0}, \varphi_{0}, 0\right)-\frac{1}{2} \nabla_{\theta, \varphi}^{2} P+U(\Omega) P=\delta(N) \delta\left(\Omega-\Omega_{0}\right),
$$


where $U(\Omega)=i \mathbf{k} \Omega=i k \cos (\theta)$, and $k$ is measured in units of $l_{p}^{-1}$. As it is well-known from the propagator method [16] the latter can be written as an integral equation as follows

$$
P\left(\Omega, N ; \Omega_{0}, 0\right)=P_{0}\left(\Omega, N ; \Omega_{0}, 0\right)-\int_{0}^{N} d s \int d \Omega^{\prime} P_{0}\left(\Omega, N ; \Omega^{\prime}, s\right) U\left(\Omega^{\prime}\right) P\left(\Omega^{\prime}, s ; \Omega_{0}, 0\right) .
$$

Eqs.(35) describes also the Euclidean rigid quantum rotator in an external field. The iteration of the latter generates the perturbation expansion of $P$ in powers of the potential $U(\Omega)$. The Fourier transform of the distribution function of the end-to-end distance of the polymer is obtained from $P\left(\Omega, N ; \Omega_{0}, 0\right)$ by integrating the latter over $\Omega$ and $\Omega_{0}$ : $G(k, N)=\int d \Omega \int d \Omega_{0} P\left(\Omega, N ; \Omega_{0}, 0\right)$. The factor $4 \pi$ is extracted from $P\left(\Omega, N ; \Omega_{0}, 0\right)$. Eq. (5) with fixed $\Omega$ and $\Omega_{0}$ is the distribution function of the end-to end distance with fixed tangents of the polymer ends. The coefficient in front of $\left(k^{2}\right)^{n}$ of the expansion of $G(k, p)$ in powers of $k^{2}$, multiplied by the factor $(-1)^{n} \Gamma(2 n+2)$ is the Laplace transform of the moment $\left\langle r^{2 n}\right\rangle$ of the end-to-end distribution function. Due to the folding character of the integrations over the contour lengths in (5), the Laplace transform of (3) with respect to $N$ permits to get rid of integrations over the contour length. Similar to the propagator method used elsewhere [16] each order of the perturbation expansion can be represented by graphs, examples of which are schematically shown in Fig.1. Each piece of the continuous

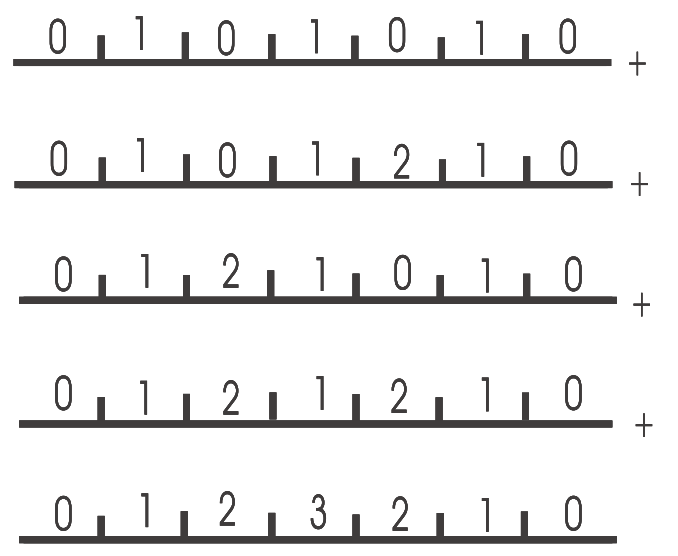

a)
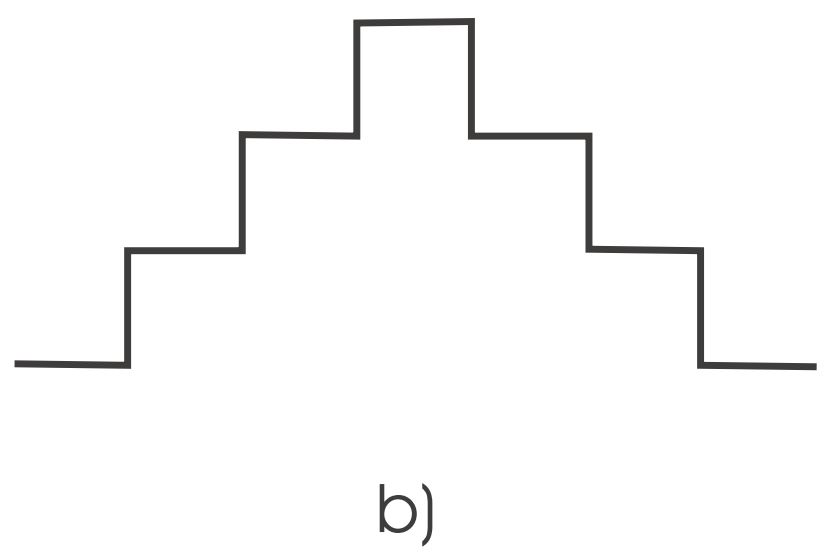

Fig. 1 - a) The 6 th order of the perturbation expansion of the end-to-end distribution function. b) Random walk with constraint associated with the last line in a).

line between two consecutive vertices are associated with the Laplace transform of the bare Green's function given by Eq.(3). The integral associated with each vertex is $\langle\cos \theta\rangle_{l^{\prime}, l ; m^{\prime}, m}=$ $\int d \Omega Y_{l^{\prime}, m^{\prime}}^{*}(\theta, \varphi) \cos \theta Y_{l, m}(\theta, \varphi)$. Using the property of the spherical functions $\cos \theta Y_{l, m}=$ $\sqrt{\left((l+1)^{2}-m^{2}\right) /\left(4(l+1)^{2}-1\right)} Y_{l+1, m}+\sqrt{\left(l^{2}-m^{2}\right) /\left(4 l^{2}-1\right)} Y_{l-1, m}$ gives $\langle\cos \theta\rangle_{l^{\prime}, l ; m^{\prime}, m}=$ $\sqrt{\left((l+1)^{2}-m^{2}\right) /\left(4(l+1)^{2}-1\right)} \delta_{l^{\prime}, l+1} \delta_{m^{\prime}, m}+\sqrt{\left(l^{2}-m^{2}\right) /\left(4 l^{2}-1\right)} \delta_{l^{\prime}, l-1} \delta_{m^{\prime}, m}$.

Due to the integrations over $\Omega$ and $\Omega_{0}$ the quantum numbers $l$ and $m$ associated with the transition probabilities (amplitudes in the quantum mechanical counterpart of the problem) 
at graph ends are equal to zero. The averages over $\cos \theta$ in each vertex gives that the quantum numbers $l$ on both sides of a vertex differ by one. The weights associated with the vertices are given by $w_{l}=\langle\cos \theta\rangle_{l, l-1}=\langle\cos \theta\rangle_{l-1, l}=l / \sqrt{4 l^{2}-1}$. The Laplace transform of the weights associated with each piece of the continuous line between two vertices, which are given by the exponential factor in Eq.(3), are given by $e_{l}=1 /(p+l(l+1) / 2)$, where $p$ is the Laplace conjugate to $N$. The quantum number $m$ is conserved along the path and is zero due to the integration over the angles at the ends. Thus, the problem of counting different paths contributing to the transition probability $\int d \Omega \int d \Omega_{0} P\left(\Omega, N ; \Omega_{0}, 0\right)$ can be formulated as a problem of a random walk consisting of odd number of steps with the constraint that the step heights are equal to one. The example of such a random walk is given in Fig.1b. The computation of the coefficients in front of $\left(k^{2}\right)^{n}$ of the expansion of $G(k, p)$ in powers of $k^{2}$ is thus related to the enumeration of configurations of such a random walk consisting of $2 n+1$ steps. This kind of the problem is closely related to the calculation of the dimensions of the irreducible representations of the Temperley-Lieb algebra [17]. One can write the expansion of $G(k, p)$ as follows

$$
G(k, p)=\left[d+\sum_{n=1}^{\infty}\left(-k^{2}\right)^{n} A^{2 n}\right]_{1,1}=\frac{1}{p}\left[I+k^{2} A^{2}\right]_{1,1}^{-1},
$$

where the last term is the result of the summation of the series, $A=d . m$ is the product of the infinite rank matrices $d$ and $m$, which are defined as follows $d_{i, j}=e_{i-1} \delta_{i, j}$ and $m_{i, j}=$ $w_{i-1} \delta_{i, j+1}+w_{i} \delta_{i, j-1}(i, j=1,2, \ldots)$. Approximating the last term in Eq.(6) by the matrices of finite rank $n$ gives an approximate formula for the distribution function, $G_{2 n-2}(k, p)$, which gives exactly the first $2 n-2$ moments and approximate expressions for higher moments where the intermediate states with $l=0,1, \ldots, n-1$ are taken into account. No matrix inversion is required for the exact Laplace transforms of the moments which may be calculated directly by the Fourier transform of (6). From the expansion in $k$ one realizes that only the $(1,1)$-element of the $2 n$-power of $A$ needs to be calculated for $\left\langle r^{2 n}\right\rangle$. Because of the simple band structure of $A$ this is obtained exactly from a truncated matrix $\tilde{A}$ of rank $n+1$.

The distribution function $G_{2 n}(k, p)(2 n$ is the number of the exact first moments contained in $\left.G_{2 n}(k, p)\right)$ can be written as

$$
G_{2 n}(k, p)=\frac{1}{p} \frac{1+f_{2}+f_{3}+\ldots+f_{n}}{1+f_{1}+f_{2}+f_{3}+\ldots+f_{n}}
$$

where $f_{k}$, which are defined for each $n$, obey the following recursion relation $f_{k}=u_{k}(1+$ $\sum_{l=k+2}^{n} f_{l}$ ), and $u_{i}=k^{2} e_{i-1} w_{i}^{2} e_{i}$. Eqs.(6-8) are exact and are the main result of the present Letter. The off diagonal matrix elements of the matrix $\left[I+k^{2} A^{2}\right]^{-1}$ give the transition probabilities from the initial state $\left(l_{a}, m_{a}=0\right)$ to the final state $\left(l_{b}, m_{b}=0\right)$. The computation of the distribution function of the end-to-end distance with fixed tangents of the end vectors demands consideration of paths with arbitrary $l_{a}, m_{a}$ and $l_{b}, m_{b}$. The consideration of the transition probability with nonzero number $m$ demands the change of the definition of the weights $w_{l}$. The simple band structure of the matrix, however, remains unchanged.

We have computed $G_{m}(k, p)$ explicitly for $m \leq 26$. The distribution function, which takes into account exact 6 moments, is given by

$$
G_{6}(k, p)=\frac{5}{3} \frac{39 k^{2}+11 k^{2} p+21 p^{3}+210 p^{2}+378+567 p}{35 p^{4}+350 p^{3}+30 k^{2} p^{2}+945 p^{2}+630 p+170 k^{2} p+3 k^{4}+210 k^{2}} .
$$


As an example we give below the 10th moment

$$
\begin{aligned}
<\left(r^{2}\right)^{5}>= & -\frac{256}{540225} N e^{-10 N}-\frac{1024}{3781575} e^{-10 N}+\frac{1408}{50625} N^{2} e^{-6 N}+\frac{15616}{455625} N e^{-6 N}- \\
& \frac{15872}{20503125} e^{-6 N}-\frac{7040}{3969} N^{3} e^{-3 N}+\frac{14080}{9261} N^{2} e^{-3 N}+\frac{1303040}{21609} N e^{-3 N}+ \\
& \frac{1285273600}{12252303} e^{-3 N}+\frac{22176}{25} N^{4} e^{-N}+\frac{4549248}{125} N^{3} e^{-N}+\frac{2338481024}{4375} N^{2} e^{-N}+ \\
& \frac{973888866304}{275625} N e^{-N}+\frac{88900199815424}{9646875} e^{-N}+\frac{256}{56260575} e^{-15 N}+\frac{12320}{9} N^{5}- \\
& \frac{726880}{27} N^{4}+\frac{21670528}{81} N^{3}-\frac{651701248}{405} N^{2}+\frac{34520038912}{6075} N- \\
& \frac{167953309696}{18225} .
\end{aligned}
$$

All 26 moments we have computed are obtained in a form similar to $\left\langle\left(r^{2}\right)^{5}>\right.$ with numerical coefficients given as fractional numbers. All moments fulfil the expected limiting behaviour for small and large $N$.

Performing the inverse Laplace transform of $G(k, p)$ by using Maple enables one to plot $G(k, N)$. In the limit of small $N$ the distribution function $G_{m}(r, N)$ approaches the rod limit $G_{\text {rod }}(r, N)=1 /\left(4 \pi N^{2}\right) \delta(r-N)$, which has the Fourier transform $G_{\text {rod }}(k, N)=\sin (k N) / k N$. In order to illustrate the accuracy of the truncated dustribution function a comparison of $G(k, N)$ with the rod limit is shown in Fig.2. The increase of $m$ extends the interval of $k$ of the

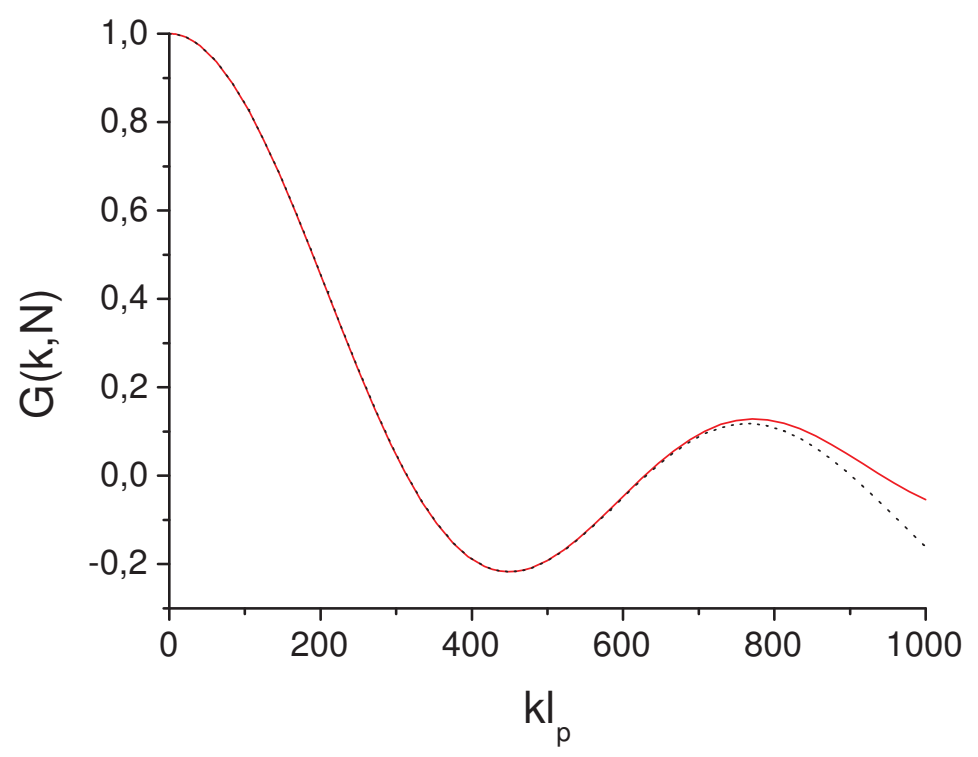

Fig. 2 - Computation of $G(k, N)$ for $N=0.01$ : Continuous: $G_{r o d}(k, N)$, dots: $G_{10}(k, N)$

agreement between $G_{m}(k, N)$ and $G_{r o d}(k, N)$. However, for large $m$ the Maple computation of the inverse Laplace transform of $G(k, p)$ becomes unstable for small $k$ at very small $N$. 
EUROPHYSICS LETTERS

The inverse Laplace transform of $G(k, p) / p^{2}$ multiplied with the factor $2 / N$ gives the structure factor of the semiflexible polymer $S(k, N)$. Performing the inverse Laplace transform by using Maple permits to get plots of the structure factor.

In conclusion, we have studied the end-to-end distribution function of a worm like chain, the problem which is equivalent to the Euclidean quantum rigid rotator in a homogeneous external field, by using the propagator method. We have established that the combinatorial problem of counting the paths contributing to the end-to-end distribution function can be mapped to the problem of random walks with constraints, which is closely related to the Temperley-Lieb algebra. Using this mapping we derived an exact expression for the FourierLaplace transform of the end-to-end polymer distance as the matrix element of the inverse of an infinite rank matrix. Starting with this result we have also derived a recursion relation permitting to compute $G(k, p)$ directly. The mapping to the problem of restricted random walks and the Temperley-Lieb algebra, which is not restricted to the computation of $G(k, N)$, gives a new tool of treating the semiflexible polymers. Eqs.(6-1) give also the exact solution for the transition amplitude of a rigid quantum rotator in an external homogeneous imaginary field in Euclidean sector.

S.S. acknowledges a support from the Deutsche Forschungsgemeinschaft (SFB 418 and Ste 981-1/1).

\section{REFERENCES}

[1] Edwards S. F., Proc. Phys. Soc. 85 (1965) 613.

[2] de Gennes P. G., Scaling Concepts in Polymer Physics (Cornell University Press, Ithaca, 1979).

[3] des Cloizeaux J. and Jannink G., Polymers in solution, Their modeling, and structure (Oxford University Press, 1990).

[4] Doi M. and Edwards S. F., The Theory of Polymer Dynamics (Clarendon Press, Oxford, 1986).

[5] Sackmann E., Macrom. Chem. Phys. 195 (1994) 7.

[6] Liverpool T. B. and Edwards S. F., Phys. Rev. Lett. 75 (1995) 3016; Liverpool T. B., Ball R. C., and Edwards S. F., Europhys. Lett. 30 (1995) 181.

[7] Kratky O., and Porod G., Recl. Trav. Chim. Pays-Bas 68 (1949) 1106.

[8] Hermans J. J. and Ullman R., Physica 18 (1952) 951.

[9] Heine S., Kratky O., and Roppert J., Makromol. Chem. 56 (1962) 150.

[10] Saito N., Takahashi K., and Yunoki Y., J. Phys. Soc. Jpn. 22 (1967) 219.

[11] Yamakawa H., Helical Wormlike Chains in Polymer Solutions, (Springer, Berlin, 1997).

[12] Wilhelm J. and Frey E., Phys. Rev. Lett. 77 (1996) 2581.

[13] Bhattacharjee J. K. , Thirumalai D., and Bryngelson J. G., cond-mat/9709345.

[14] Samuel J. and Sinha S., cond-mat/0203483, submitted to Phys. Rev. E.

[15] Zinn-Justin J., Quantum Field Theory and Critical Phenomena (Clarendon Press, Oxford, 1996). Chap.3.

[16] Feynman R. P. and Hibbs A. R., Quantum Mechanics and Path Integrals (MvGraw-Hill, New York, 1965).

[17] Temperley H. N. V. and Lieb E. H., Proc. Roy. Soc. London, A 322 (1971) 251.

[18] Stepanow S., J. Chem. Phys., 115 (2001) 1565. 